\title{
Moving Bed Drying Method
}

National Cancer Institute

\section{Source}

National Cancer Institute. Moving Bed Drying Method. NCI Thesaurus. Code C112981.

A process that removes water or volatile solvents from an agitated or tumbling bed of solids by relying on heat transfer from the equipment surface or a hot gas to the solid sample. 